\title{
Initial Report of a New Suture Cerclage Tape for Sternal Closure
}

\section{Paul DiGiorgi ${ }^{1}$}

${ }^{1}$ Lee Health

April 21, 2021

\begin{abstract}
PURPOSE: We sought to evaluate a new, flexible suture cerclage tape for sternal closure after cardiac surgery. DESCRIPTION: Fiber Tape (Arthrex, Naples, FL, USA) suture cerclage has been successfully utilized in the stabilization of long bone fractures. For sternal closure, it is placed similarly to wire cerclage. Uniquely, it allows a measurable amount of tension to be applied to the sternum and it is at least twice as strong as stainless steel wire. We hypothesized this new flexible tape would provide short term safety and efficacy, as measured by incidence of deep sternal wound infection. EVALUATION: We performed a retrospective, single center analysis of adult patients undergoing sternotomy closure with FT. The observed incidence of deep sternal wound infection (DSWI) was compared to the expected incidence calculated through the Society of Thoracic Surgery (STS) risk calculator. A total of 45 patients were closed with FT. No patients suffered DSWI. Thirty of the 45 patients had STS risk calculations. For these 30 patients, the expected rate of DSWI was $0.2 \%$. CONCLUSIONS: Despite study limitations, FT appears to be a safe method for sternal closure.
\end{abstract}

Initial Report of a New Suture Cerclage Tape for Sternal Closure

Paul L. DiGiorgi, MD, FACS

Cardiothoracic Surgeon

Shipley Cardiothoracic Center

Lea Health

Acknowledgements: We acknowledge Ms. Nalani Yeager, RN for her assistance in data collection and analysis.

Funding statement: no funding was provided to our institution to perform this study.

Dr. DiGiorgi is a consultant for Arthrex, Inc.

This study has not been presented elsewhere.

Contact information for corresponding author:

Paul DiGiorgi MD, FACS

Shipley Cardiothoracic Center

9981 South HealthPark Drive, suite 156

Fort Myers, FL 33908

(239) 343-6341

pdigiorgi@earthlink.net

Abstract 
PURPOSE: We sought to evaluate a new, flexible suture cerclage tape for sternal closure after cardiac surgery.

DESCRIPTION: Fiber Tape (Arthrex, Naples, FL, USA) suture cerclage has been successfully utilized in the stabilization of long bone fractures for several years. To date, it has not been reported in sternal closure. For sternal closure, it is placed similarly to wire cerclage. Uniquely, it allows a measurable amount of tension to be applied to the sternum and it is at least twice as strong as stainless steel wire. We hypothesized this flexible tape would provide short term safety and efficacy, as measured by incidence of deep sternal wound infection.

EVALUATION: We performed a retrospective, single center analysis of adult patients undergoing sternotomy closure with FT. The observed incidence of deep sternal wound infection (DSWI) was compared to the expected incidence calculated through the Society of Thoracic Surgery (STS) risk calculator. A total of 45 patients were closed with FT. No patients suffered DSWI. Thirty of the 45 patients had STS risk calculations. For these 30 patients, the expected rate of DSWI was $0.2 \%$.

CONCLUSIONS: Despite study limitations, FT appears to be a safe method for sternal closure.

\section{TECHNOLOGY}

Stainless steel wire cerclage (SSC) is the most common method of closing the sternum in adult cardiac surgery. Despite relatively low cost and familiarity, stainless wire closure remains vulnerable to fracturing, uneven force application, boney pull through, and sharp edges that can lead to glove perforation with wound contamination and injury to staff. These limitations are associated with excessive boney movement, increased postoperative pain, nonunion, reoperation, and potentially life threatening deep sternal wound infection the latter two effect thousands of patients each year in the United States alone[1]. Given these potential limitations, boney reconstruction throughout the body has generally progressed beyond stainless steel wires.

Attempts at improving on SSC have been made over time. Other devices to close the sternum with improved outcomes have been developed[2-8]. Unfortunately, each has suffered from cost limitations, complexity, study selection bias, and/or ineffectiveness. Additionally, clinical outcomes did not necessarily reflect contemporary experience and possibly introduce new complications[9]. As a result, SSC remains the most common method to close the sternum despite its limitations.

More recently, a flexible suture cerclage tape (Fiber Tape, Arthrex, Inc., Naples, FL, USA) has been successfully utilized in the stabilization of bone fractures [10]. Fiber Tape (FT) is flexible avoiding potential glove penetration of wire ends and easier to work with compared to much stiffer stainless steel wire. FT is significantly stronger than stainless steel wire as well $(2-4 \mathrm{x}$, internal report). It is placed similarly to SSC with standard interrupted or figure-of-eight suturing techniques simplifying closure compared to plates. Unlike other devices, it allows a measurable amount of tension to be applied using a tensioner thereby allowing optimal tension to be applied to the sternum.

We hypothesized FT would provide short term safety and efficacy similar to SSC in adult cardiac surgery patients.

\section{TECHNIQUE}

FT closure is performed in a similar manner to SSC. The manubrium is closed with two interrupted tapes. Then, figure of eight tape placement is performed around the sternal body in separate interspaces (Figure 1). The tape ends are then brought through a preformed loop and hand tightened down closing the sternum loosely. A tensioner is then placed on each tape and gradually tensioned (Figure 2) to 60-80 newtons. Once adequate tension across all tapes is achieved (Figure 3), the tape ends are then cut (Figure 4).

\section{CLINICAL EXPERIENCE}

We performed a retrospective, single center trial for adult patients undergoing sternotomy closure after 
cardiac surgery. We measured clinical outcomes of our initial cohort of adult cardiac surgical patients closed with FT cerclage at HealthPark Medical Center (Fort Myers, FL, USA) between March 2019 and September 2019. Patients' outcomes were quantified and compared to expected results based on national data calculated through the Society of Thoracic Surgery (STS) risk calculator. Patient preoperative characteristics are summarized in Table 1 . There were 45 patients with an average age of 70 years old. Operative date are summarized in Table 2. Relevant postoperative outcomes are summarized in Table 3. The observed incidence of deep sternal wound infection (DSWI) was compared to the expected incidence calculated through the Society of Thoracic Surgery (STS) risk calculator. A total of 45 patients were closed with FT. No patients suffered DSWI. Thirty of the 45 patients had STS risk calculations. For these 30 patients, the expected rate of DSWI was $0.2 \%$. No patients underwent reoperation for any reason. The STS expected incidence of reoperation was $3.2 \%$. After a short learning curve we found that FT closure was faster than a typical SSC closure as well.

\section{COMMENT}

Sternal closure continues to be performed most commonly with SSC despite mulitple attempts at creating improved methods and materials. More recently, FT has been shown to be an effective material for boney reconstruction [10]. It is significantly stronger than stainless steel and handles much more easily being a braided material. Because it is placed in a similar manner to SSC the learning curve is steep. Additionally, as a cerclage closure, it allows circumferenctial closure of the bone. Our initial experience was positive: we found no early failures, it was faster after a few cases, allowed exact tension to be applied to the bone, and there were no deep (or superficial) sternal wound infections found. There are several limitations to our study however. This is an initial cohort study and not randomized. With only 45 patients it is likely underpowered to definitively know its effect on deep sternal wound infection rates. Other criteria for efficacy should also be evaluated and were not in the scope of this reoprt. These include pain and healing assessments as well as cost effectiveness. These will be part of a more comprehensive, randomzied trial.

Acknowledgements

Acknowledgements: We acknowledge Ms. Nalani Yeager, RN for her assistance in data collection and analysis.

Disclosures

Dr. DiGiorgi receives consultant fee from Arthrex, Inc.

\section{FIGURE 1.}

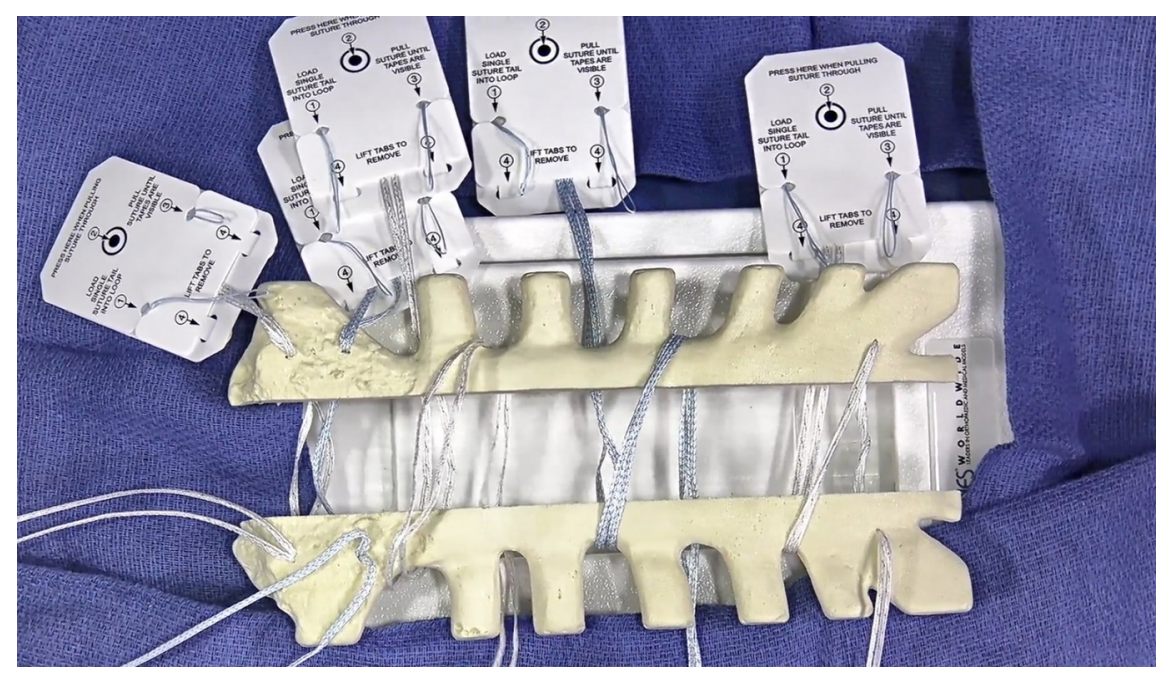


FIGURE 2.

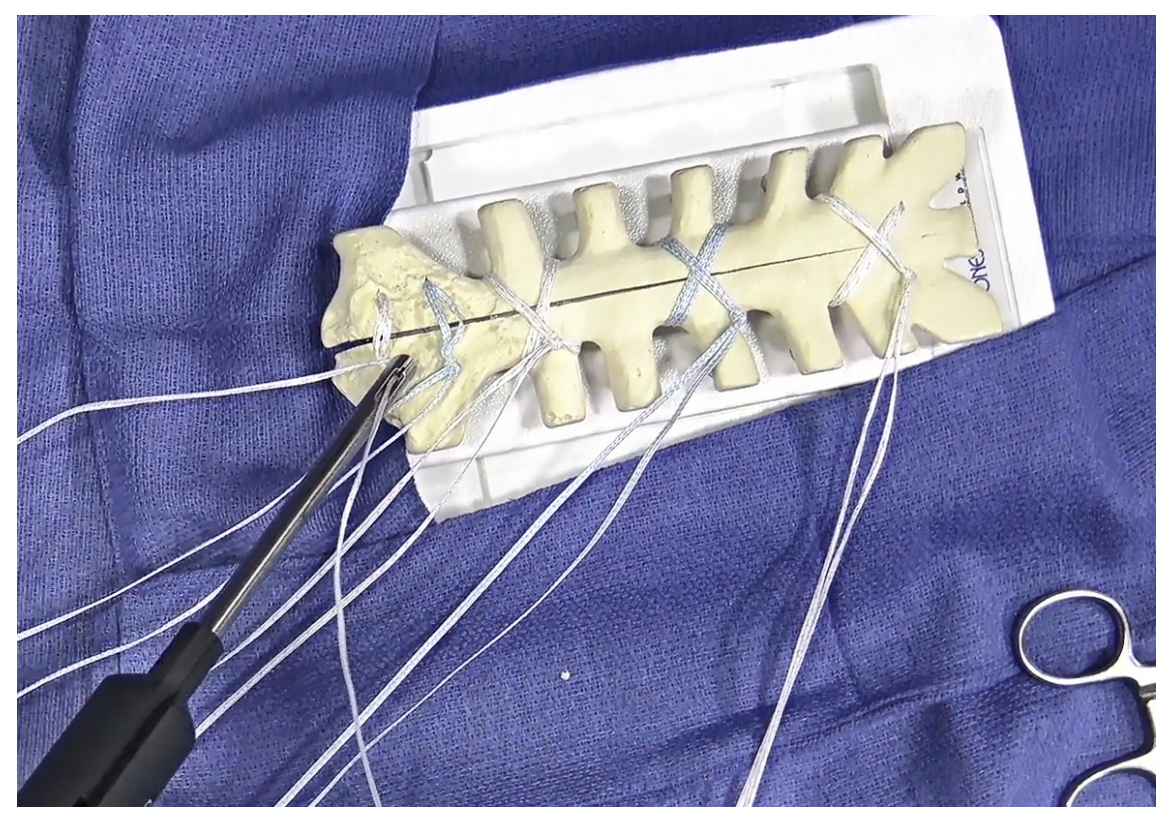

FIGURE 3.

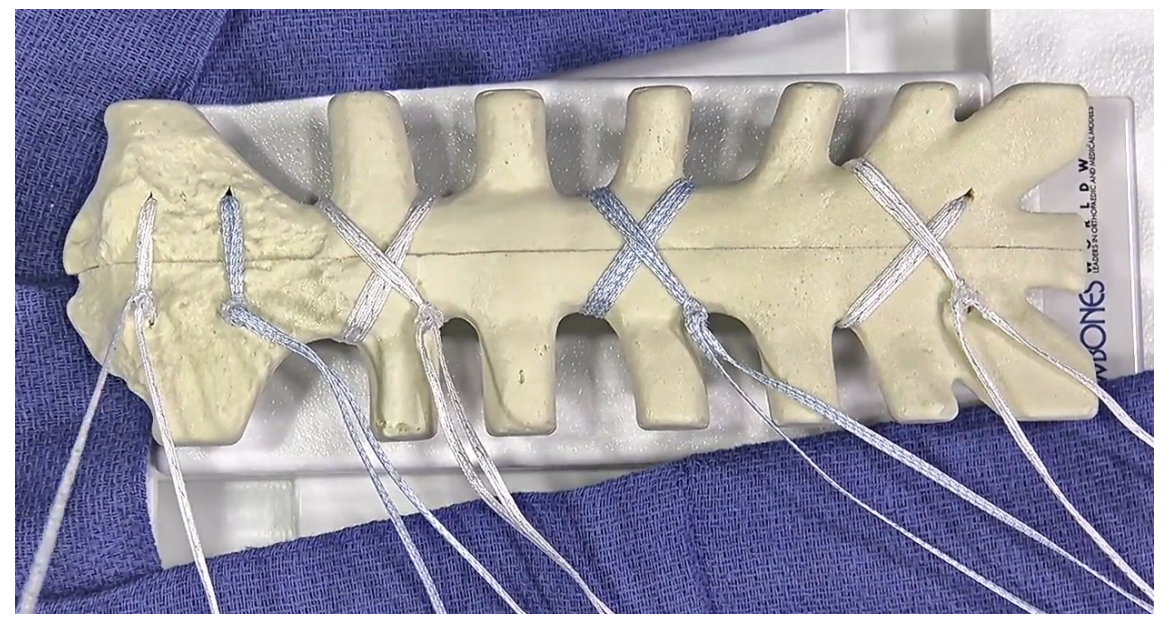

FIGURE 4. 


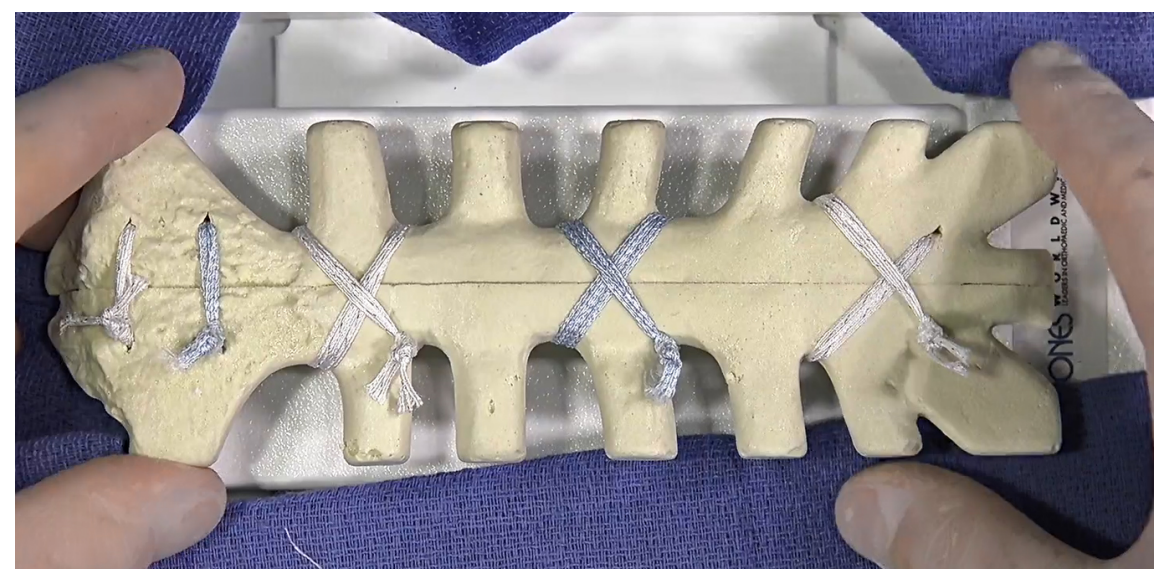

References

1. D'Agostino, R.S., et al., The Society of Thoracic Surgeons Adult Cardiac Surgery Database: 2018 Update on Outcomes and Quality.Ann Thorac Surg, 2018. 105 (1): p. 15-23.

2. Boustany, A.N., P. Ghareeb, and K. Lee, Prospective, randomized, single blinded pilot study of a new FlatWire based sternal closure system. J Cardiothorac Surg, 2014. 9 : p. 97.

3. Bejko, J., et al., Nitinol flexigrip sternal closure system and standard sternal steel wiring: insight from a matched comparative analysis. J Cardiovasc Med (Hagerstown), 2015. 16 (2): p. 134-8.

4. Graham, G., et al., Randomized Trial of Sternal Closure for Low Risk Patients: Rigid Fixation versus Wire Closure. Heart Surg Forum, 2017. 20 (4): p. E164-E169.

5. Imagawa, H., et al., A prospective randomized study of sternal closure: comparison of Mersilene tape versus standard wire closure. Ann Thorac Cardiovasc Surg, 2004. 10 (6): p. 362-6.

6. Leinberger, T., et al., The COSTA Study: Sternal Closure in High-Risk Patients - A Prospective Randomized Multicenter Trial. Thorac Cardiovasc Surg, 2018.

7. Melly, L., et al., A new cable-tie-based sternal closure device: infectious considerations. Interact Cardiovasc Thorac Surg, 2013. 17 (2): p. 219-23; discussion 223-4.

8. Allen, K.B., et al., Randomized, multicenter trial comparing sternotomy closure with rigid plate fixation to wire cerclage. J Thorac Cardiovasc Surg, 2017. 153 (4): p. 888-896.e1.

9. Samuels, L., Sternal Closure With Tie Bands: A Word of Caution. Ann Thorac Surg, 2016. 102 (2): p. e121-2.

10. Eyberg, B.A., et al., Suture cerclage for stabilizing the humeral shaft during shoulder arthroplasty. JSES Int, 2020.4 (3): p. 688-693.

Tables

Table 1. Preoperative characteristics of FT patients.

\section{All Fiber Tape Patients}

Preoperative Characteristics

Number of patients

Average Age (years)

Male (n, percent)

Mean BMI

$\begin{array}{ll}\text { Preoperative Characteristics } & \text { Preoperative Characteristics } \\ 45 & - \\ 70 & - \\ 33 & 73.3 \% \\ 27.1 & -\end{array}$




$\begin{array}{lll}\text { Mean Height (cm) } & 172.9 & - \\ \text { Mean Weight (kg) } & 81.4 & - \\ \text { Race - Caucasian (n, percent) } & 41 & 91.1 \% \\ \text { Race - African American (n, percent) } & 3 & 6.7 \% \\ \text { Hispanic Ethnicity (n, percent) } & 3 & 6.7 \% \\ \text { Diabetes (n, percent) } & 15 & 33.3 \% \\ \text { Renal Failure (n, percent) } & 0 & 0.0 \% \\ \text { Chronic lung disease (n, percent) } & 20 & 44.4 \% \\ \text { Mild } & 10 & 22 \% \\ \text { Moderate } & 1 & 2.2 \% \\ \text { Severe } & 4 & 8.9 \% \\ \text { Severity unknown } & 5 & 11.1 \% \\ \text { Current tobacco use (n, percent) } & 3 & 6.7 \% \\ \text { Former tobacco use (n, percent) } & 27 & 60.0 \% \\ \text { Never smoker (n, percent) } & 15 & 33.3 \% \\ \text { Home oxygen use (n, percent) } & 0 & 0.0 \% \\ \text { Pneumonia, remote (n, percent) } & 6 & 13.3 \% \\ \text { Illicit drug use, remote (n, percent) } & 1 & 2.2 \% \\ \text { Liver Disease (n, percent) } & 1 & 2.2 \% \\ \text { Immunocompromised (n, percent) } & 4 & 8.9 \% \\ \text { Mediastinal radiation (n, percent) } & 1 & 2.2 \% \\ \text { Prior sternotomy (n, percent) } & 5 & 11.1 \% \\ \text { Peripheral vascular disease (n, percent) } & 11 & 24.4 \% \\ \text { CAD (n, percent) } & 39 & 86.7 \% \\ \text { LVEF (AVG) } & 55 & -\end{array}$

Table 2. Intraoperative data for FT patients.

\section{Operative Data}

\begin{tabular}{lll}
\hline Isolated CABG (n, percent) & 22 & $48.9 \%$ \\
Isolated Valve (n, percent) & 1 & $2.2 \%$ \\
CABG/Valve (n, percent) & 7 & $16 \%$ \\
Other (n, percent) & 15 & $33 \%$ \\
CPB time, average (minutes) & 90 & - \\
XCL time, average (minutes) & 67 & - \\
Operative time, average (minutes) & 287 & - \\
\hline
\end{tabular}

Table 3. Outcomes data for FT patients.

\begin{tabular}{lllll}
\hline Postop Events & n & Observed & Expected & O/E Ratio \\
\hline Any reoperation & 0 & $0.0 \%$ & $3.2 \%$ & 0.00 \\
Deep sternal wound infection & 0 & $0.0 \%$ & $0.2 \%$ & 0.00 \\
\hline
\end{tabular}

Figure Legends

Figure 1. Placement of Fiber Tape around the sternum. All sutures are placed loosely first then tensioned. Two interrupted sutures are placed in the manubrium followed by three figure of eight sutures along the sternal body. 
Figure 2. After passing the sutures through a preformed knot and hand tightening a tensioner sets the desired tension of each Fiber Tape.

Figure 3. All Fiber Tapes tensioned down before final knot throws and triming.

Figure 4. Final closure with knots trimmed. 